\title{
A study of the EB-type eclipsing binary GR Tauri with mass transfer
}

\author{
Sheng-hong Gu ${ }^{1,2}$, Pei-sheng Chen ${ }^{1,2}$, Yu-kou Choy ${ }^{2}$, Kam-cheung Leung ${ }^{3}$, \\ Wai-keung Chung ${ }^{3}$, and Tak-sun Poon ${ }^{3}$ \\ 1 National Astronomical Observatories/Yunnan Observatory, Joint laboratory for optical astronomy, \\ Chinese Academy of Sciences, Kunming, PR China \\ e-mail: shenggu@public.km.yn.cn \\ 2 Ho Koon Nature Education cum Astronomical Centre, Sik Sik Yuen, Hong Kong, PR China \\ ${ }^{3}$ Hong Kong Astronomical Society, Hong Kong, PR China
}

Received 21 December 2001 / Accepted 10 May 2004

\begin{abstract}
In this paper, new CCD $B V$ light curves of the EB-type eclipsing binary GR Tau, which were obtained in 1999, are analyzed by means of the Wilson-Devinney program. The photometric solution of this system is obtained, and its absolute parameters are also derived. Our study has demonstrated that GR Tau is a near-contact binary system with an almost-contact semidetached configuration in which the primary fills its Roche lobe, and both components are main-sequence stars. The asymmetric shape of the light curves can be explained by a hot spot on the surface of the secondary, which is created by the mass transfer from the primary to the secondary. GR Tau belongs to the V1010 Oph subclass of near-contact binaries, and is a good example of a system in the broken-contact phase predicted by the TRO theory.
\end{abstract}

Key words. stars: binaries: close - stars: individual: GR Tau

\section{Introduction}

GR Tau is a very interesting close binary system which has attracted some attention (Yamasaki et al. 1984; Hanzl 1990; Fang et al. 1994; Lázaro et al. 1995; Zhang et al. 2002). In these papers, GR Tau is found to exhibit an active feature viz. the light curves vary between being symmetric and being asymmetric on a short time scale. When the light curve is asymmetric, there is an O'Connell effect, namely the first light maximum at phase 0.25 is brighter than the second at phase 0.75 . This phenomenon is frequent in near-contact binaries (Shaw 1990, 1994). In addition to this effect, both eclipses are deeper than in the symmetric light curves.

In this paper, our new $B V$ light curves of GR Tau are analyzed using the Wilson-Devinney code (Wilson \& Devinney 1971; Wilson 1992). A reasonable explanation for the O'Connell effect of GR Tau is given, which is based on the gas stream from the primary brightening the trailing hemisphere of the secondary. This can be inferred from the semidetached configuration of GR Tau where the primary fills its Roche lobe, and from the decreasing period of the binary system.

\section{Observations}

The new observations for GR Tau were carried out using the $0.5 \mathrm{~m}$ telescope at the Ho Koon Astronomical Centre of Hong Kong on November 14, 15, 17 and December 7, 1999.
A ST-8 CCD camera with a $1530 \times 1020$ pixel chip was used. The observations were made through standard $B$ and $V$ filters. The integration time was 40 to $120 \mathrm{~s}$, depending on the weather condition and the zenith distance of the star. The cycle length of one set of $B$ and $V$ observations was about 8 to $12 \mathrm{~min}$.

All observed CCD images were reduced by means of the IRAF package in the standard fashion. In the CCD frame, a star with a similar brightness to GR Tau was chosen as comparison. The magnitude values of GR Tau, comparison star, and other reference stars were determined using the APPHOT subpackage of IRAF. No light variation was found for this comparison star during the observations. 132 and 129 individual points were obtained in $B$ and $V$ bands, respectively.

From our observations, a primary minimum time is determined. To calculate a new ephemeris for GR Tau, all published photoelectric minimum times were collected from the literature and analyzed using the linear least-squares method. There is considerable scatter in the $(\mathrm{O}-\mathrm{C})$ values; this can be attributed to the low precision of those secondary minimum times which could not be determined with good accuracy due to the asymmetric light curve and shallow secondary eclipse. Therefore, all the secondary minimum times were eliminated from the data set. The remaining primary minimum times, which are listed in Table 1, were fitted using the linear least-squares method, and a new ephemeris formula was obtained as follows:

Min. $I=\operatorname{JD}($ Hel. $) 2444$ 573.1098( \pm 0.0009$)$

$+0.42985091( \pm 0.00000008) E$. 
Table 1. The primary minimum times of GR Tau.

\begin{tabular}{|c|c|c|c|}
\hline JD(Hel.) & Cycle & $(\mathrm{O}-\mathrm{C})$ & Source \\
\hline 2444544.3075 & -67 & -.0012 & Yamasaki et al. (1984) \\
\hline 2444573.1074 & 0 & -.0013 & $"$ \\
\hline 2444578.2643 & 12 & -.0026 & $"$ \\
\hline 2444579.1252 & 14 & -.0014 & ” \\
\hline 2446414.1619 & 4283 & .0009 & Fang et al. (1994) \\
\hline 2446415.0209 & 4285 & .0002 & $"$ \\
\hline 2446438.6622 & 4340 & -.0003 & Faulkner (1986) \\
\hline 2447821.4944 & 7557 & .0009 & Wunder et al. (1992) \\
\hline 2447827.5141 & 7571 & .0027 & Hanzl (1990) \\
\hline 2447849.4363 & 7622 & .0025 & $"$ \\
\hline 2447881.6752 & 7697 & .0025 & Mullis \& Faulkner (1991) \\
\hline 2447889.4079 & 7715 & -.0021 & Hanzl (1990) \\
\hline 2447945.2940 & 7845 & .0034 & Hanzl (1991) \\
\hline 2448288.3122 & 8643 & .0004 & Agerer (1991) \\
\hline 2448619.2975 & 9413 & .0003 & Hanzl (1994) \\
\hline 2448936.5294 & 10151 & .0021 & Lazaro et al. (1995) \\
\hline 2448938.6757 & 10156 & -.0008 & ” \\
\hline 2448952.4314 & 10188 & -.0004 & ” \\
\hline 2448953.2915 & 10190 & .0000 & $"$ \\
\hline 2449334.1394 & 11076 & -.0002 & Fang et al. (1994) \\
\hline 2449734.3334 & 12007 & .0024 & Agerer \& Hubscher (1996) \\
\hline 2450422.5188 & 13608 & -.0038 & Agerer \& Hubscher (1998) \\
\hline 2451139.5145 & 15276 & .0003 & Agerer et al. (1999) \\
\hline 2451173.0380 & 15354 & -.0027 & Zhang et al. (2002) \\
\hline 2451176.0496 & 15361 & -.0001 & $"$ \\
\hline 2451198.4049 & 15413 & .0011 & Agerer et al. (1999) \\
\hline 2451486.3991 & 16083 & -.0050 & Agerer et al. (2001) \\
\hline 2451500.1586 & 16115 & -.0007 & Present study \\
\hline 2451571.0834 & 16280 & .0007 & Zhang et al. (2002) \\
\hline 2451574.0894 & 16287 & -.0022 & $"$ \\
\hline 2452211.1277 & 17769 & -.0030 & " \\
\hline 2452214.1353 & 17776 & -.0043 & ” \\
\hline 2452220.1553 & 17790 & -.0022 & $"$ \\
\hline
\end{tabular}

Based on this ephemeris, the $(\mathrm{O}-\mathrm{C})$ values of minimum times were calculated; they are listed in Table 1 . To clarify the period change for GR Tau, these $(\mathrm{O}-\mathrm{C})$ values are plotted in Fig. 1, where a decreasing period can be found. Thus, a quadratic fitting for all of primary minimum times with the least-squares method was carried out, and the following ephemeris was derived:

$$
\begin{aligned}
\text { Min. } I= & \operatorname{JD}(\text { Hel. }) 2444573.1070( \pm 0.0007) \\
& +0.42985191( \pm 0.00000019) E \\
& -5.43( \pm 0.98) \times 10^{-11} E^{2}
\end{aligned}
$$

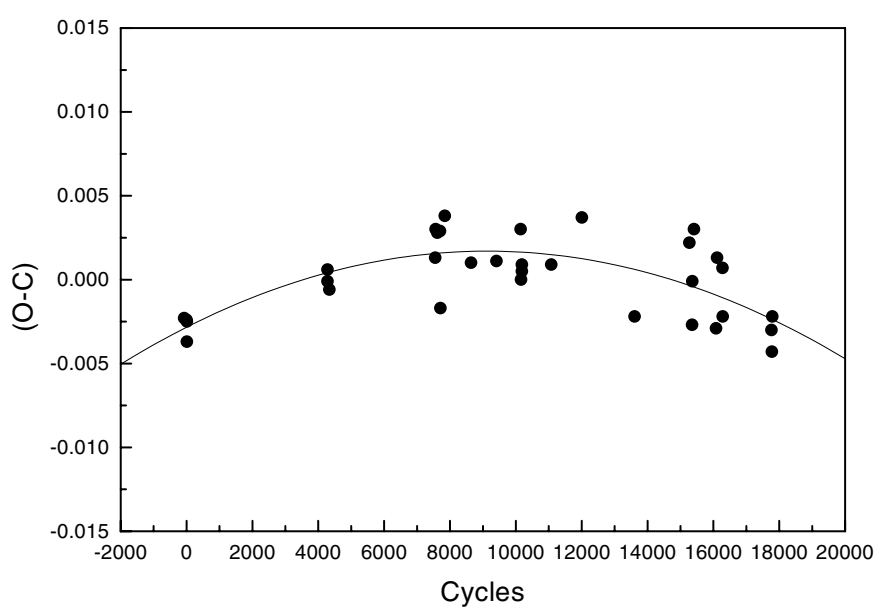

Fig. 1. The O-C diagram for the primary minimum times of GR Tau. The solid line represents the quadratic fitting.

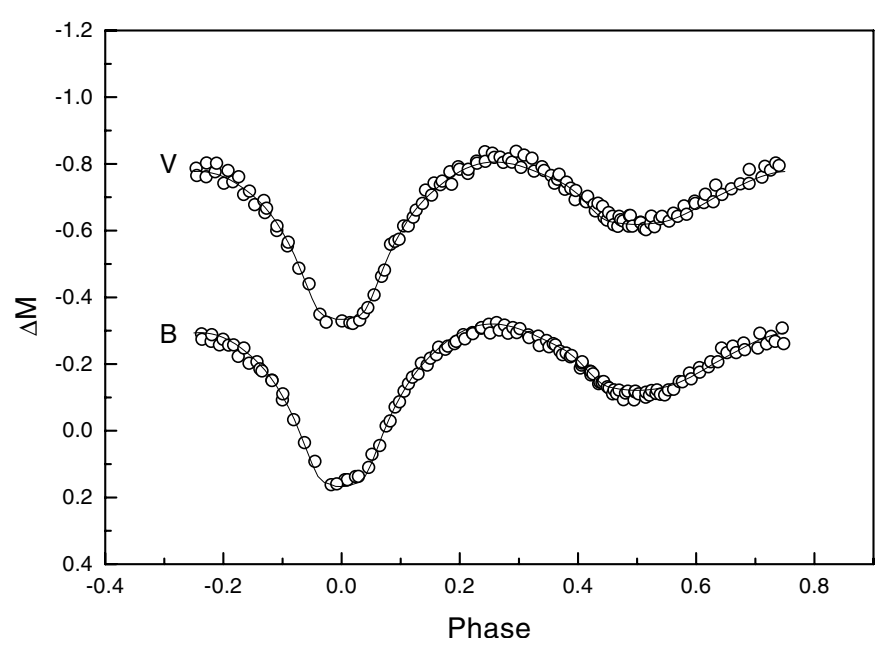

Fig. 2. The light curve of GR Tau in the $B$ and $V$ bands. The open circles and solid lines represent the observational and theoretical light curves respectively.

From formulae (2), the rate of the period decrease is $\mathrm{d} P / \mathrm{d} E=$ $-1.09 \times 10^{-10}$ days. The quadratic fitting to the $(\mathrm{O}-\mathrm{C})$ values is also displayed in Fig. 1.

For our new observation, the phases of the data points are calculated using the above formula (1), and the $B V$ light curves are displayed in Fig. 2, where $\Delta M$ represents the magnitude difference between GR Tau and the comparison star, and the open circles represent the individual observations.

\section{Light curve analysis}

In order to derive the orbital elements of GR Tau, the present CCD light curves in $B$ and $V$ bands are analyzed simultaneously by means of the 1992 version of the Wilson-Devinney program (Wilson \& Devinney 1971; Wilson 1992). The individual points in $B$ and $V$ bands are used directly for photometric analysis. As GR Tau is a single-lined spectroscopic binary, no spectroscopic mass ratio is available. The mass ratio of the system needs to be found through the usual $\Sigma-q$ method, where $\Sigma$ is the sum of the weighted squares of the residuals. Because our observed light curves exhibit asymmetry, this may affect 


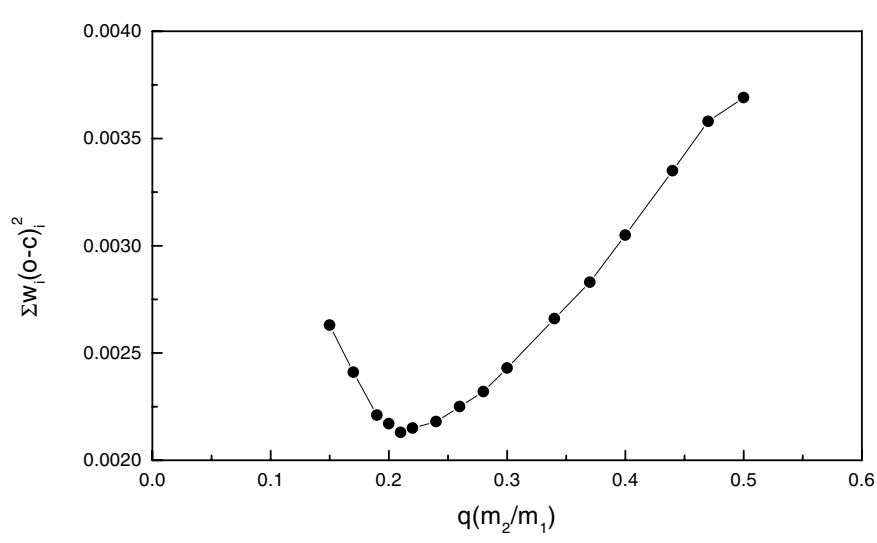

Fig. 3. The relation of $\Sigma-q$ for GR Tau.

the result for the mass ratio $q$. Therefore, we performed the calculation according to the following procedure.

In the observations made by other investigators, the light curve of GR Tau usually shows asymmetry, and the asymmetry always appears in the same phase region which is around orbital phase 0.25 . This feature implies that such a phenomenon may be caused by a hot spot on the trailing side of the secondary, which is created by the mass transfer from the primary to the secondary. This idea is supported by the fact that the period of the system is decreasing. Hence, in order to avoid that the asymmetry of the light curve affects the determination of the real mass ratio, first we estimate proper spot parameters through some test calculations using the WD program, then we carry out the procedure for finding the mass ratio. Here, the latitude of the hot spot is assumed to be 90 degrees, which means that its center is on the equator of the secondary.

The range of the mass ratio can be estimated from the results of Yamasaki et al. ( $q=0.20$ and $q=0.32$ ), Lázaro et al. $(q=0.27)$, and Zhang et al. $(q=0.22$ and $q=0.25)$. To be on the safe side, we searched a wider range. The mass ratio $q$ was fixed as $0.15,0.17,0.19,0.20,0.21,0.22,0.24,0.26,0.28$, $0.3,0.34,0.37,0.4,0.44,0.47,0.5$, respectively, and we tried to find the best value. For each assumed value of $q$, the light curves were solved by means of Wilson \& Devinney's DC program based on mode 2 (detached). In the photometric solution, the values of some parameters were adopted as follows: the temperature of the primary is $T_{1}=7500 \mathrm{~K}$ from its spectral type A9V (Lázaro et al. 1995) according to Eaton \& Poe's (1984) scale; the gravity darkening coefficients $g_{1}=1.0, g_{2}=$ 0.32 and the bolometric albedos $A_{1}=1.0, A_{2}=0.5$ are set for the primary and the secondary as usual; the limb darkening coefficients are taken from the table of Díaz-Cordovés et al. (1995). When performing the differential correction (DC) calculation, the following parameters were adjusted: the inclination $i$, the temperature of the secondary $T_{2}$, the potentials of the two components $\Omega_{1}$ and $\Omega_{2}$, the luminosity of the primary $L_{1}$, the longitude of the spot center, the radius of the spot, and the temperature factor $\left(T_{\text {spot }} / T_{\text {star }}\right)$ of the spot. After many runs, the solutions converged to the final results for all assumed mass ratios. The $\Sigma-q$ relation is illustrated in Fig. 3, where the lowest value of $\Sigma$ is found at $q=0.21$.
Table 2. The photometric solution of GR Tau.

\begin{tabular}{ll}
\hline \hline Element & Value \\
\hline$i$ & $83.353 \pm 0.195$ \\
$T_{1}$ & $7500 \mathrm{~K}$ \\
$T_{2}$ & $3434 \mathrm{~K} \pm 78 \mathrm{~K}$ \\
$g_{1}$ & 1.000 \\
$g_{2}$ & 0.320 \\
$A_{1}$ & 1.000 \\
$A_{2}$ & 0.500 \\
$\Omega_{2}$ & $2.3117 \pm 0.0135$ \\
$\Omega_{1}=\Omega_{\text {inner }}$ & 2.2798 \\
$q$ & $0.2192 \pm 0.0030$ \\
$L_{1 B} /\left(L_{1}+L_{2}\right)_{B}$ & $0.9985 \pm 0.0011$ \\
$L_{1 V} /\left(L_{1}+L_{2}\right)_{V}$ & $0.9964 \pm 0.0012$ \\
$x_{1 B}$ & 0.710 \\
$x_{2 B}$ & 0.757 \\
$x_{1 V}$ & 0.630 \\
$x_{2 V}$ & 0.733 \\
$r_{1}($ pole $)$ & $0.4803 \pm 0.0010$ \\
$r_{1}($ point $)$ & $0.6501 \pm 0.0059$ \\
$r_{1}($ side $)$ & $0.5208 \pm 0.0013$ \\
$r_{1}($ back $)$ & $0.5440 \pm 0.0012$ \\
$r_{2}$ (pole $)$ & $0.2314 \pm 0.0062$ \\
$r_{2}($ point $)$ & $0.2934 \pm 0.0246$ \\
$r_{2}($ side $)$ & $0.2399 \pm 0.0072$ \\
$r_{2}($ back $)$ & $0.2667 \pm 0.0118$ \\
Latitude $_{\text {spot }}$ & $90^{\circ}$ \\
Longitude & \\
Rpot & \\
Temperature factor $_{\text {spot }}$ & $1.50 \pm 0.04$ \\
\hline &
\end{tabular}

The result for the mass ratio shows that the most likely $q$ is between 0.21 and 0.22 , therefore, we release the mass ratio $q=m_{2} / m_{1}$ as a free parameter and continue the DC calculation based on the above initial solution with $q=0.21$. To avoid a correlation between the adjusted parameters, they were divided into three subsets $\left\{\right.$ longitude $_{\mathrm{spot}}$, radius $_{\mathrm{spot}}$, temperature factor $\left._{\mathrm{spot}}\right\},\{i\}$, and $\left\{T_{2}, \Omega_{1}, \Omega_{2}, q, L_{1}\right\}$, then each subset was adjusted separately until the three subsets converged. After some runs, the solution converged to mode 4 (semidetached), so we changed the mode from 2 to 4 and continued the DC calculation until the final photometric solution of GR Tau was derived; it is listed in Table 2. The final configuration of the system is semidetached but almost-contact with the primary filling its Roche lobe, and the secondary is very close to its Roche lobe. We used the Nightfall program (Wichmann 1998) to show the final configuration of GR Tau (Fig. 4). 

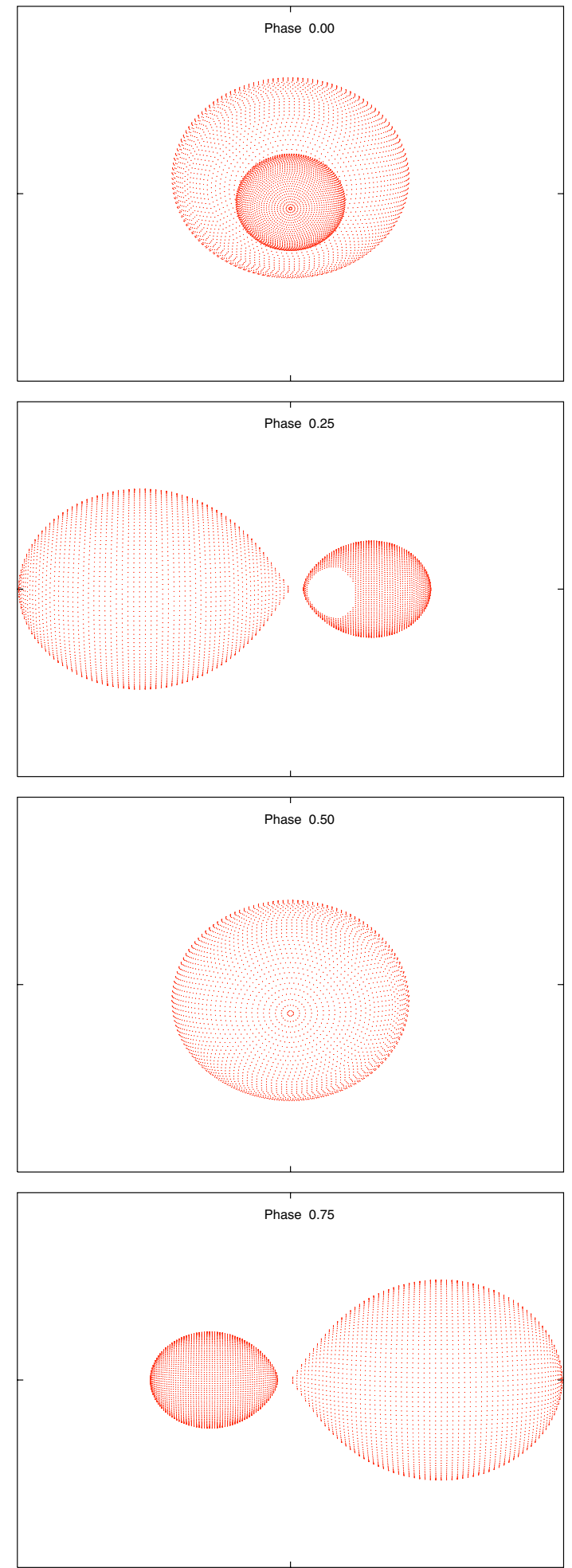

Fig. 4. The configuration of GR Tau in phase $0.0,0.25,0.5$, and 0.75 .

Now it seems very natural to add a hot spot on the secondary for such a semidetached system, because the primary fills its Roche lobe so that it can transfer mass to the secondary, and produce a hot spot (located at latitude 90 degrees) on the surface of the secondary. Further evidence for this idea is the decreasing period of the system. It can be imagined that the asymmetry of the light curve can also be reproduced if
Table 3. The absolute parameters of GR Tau.

\begin{tabular}{ll}
\hline \hline Parameter & Value \\
\hline$R_{1}$ & $1.49 \pm 0.02 R_{\odot}$ \\
$R_{2}$ & $0.71 \pm 0.04 R_{\odot}$ \\
$M_{1}$ & $1.45 \pm 0.11 M_{\odot}$ \\
$M_{2}$ & $0.32 \pm 0.02 M_{\odot}$ \\
$L_{1}$ & $6.38 \pm 0.37 L_{\odot}$ \\
$L_{2}$ & $0.06 \pm 0.01 L_{\odot}$ \\
\hline
\end{tabular}

a cool spot is put on one of the two components. However, Lázaro et al. (1995) and Zhang et al. (2002) had already refuted this idea.

By means of Wilson \& Devinney's LC program, the theoretical light curves for the above final solution are calculated, and plotted in Fig. 2, where it can be seen that the computed light curves fit the observational ones well.

\section{Discussion and conclusions}

Up to now, there are nine sets of light curves for GR Tau. Our new light curves are similar to the ones in 1980 (Yamasaki et al. 1984) and the ones in 1985, 1993, 1998, 2000, 2001 (Zhang et al. 2002), which have deeper light minima and a stronger O'Connell effects. However, the light curve in 1990 (Hanzl 1990) and the ones in 1992 (Lázaro et al. 1995) have shallower light minima and a weaker O'Connell effects. These features indicate that the light curve of GR Tau is variable.

In our new observation, the light curve distortion appears in the phase range $0.15-0.45$ so that the part of light curve around the secondary minimum is obviously asymmetric. Maximum I is brighter by about 0 . 03 than Maximum II in the $B$ band, and by about 0 . 04 in the $V$ band. The asymmetry appearing in the light curve of GR Tau may be caused by mass transfer between the two components. Our present result suggests that the system is a semidetached binary with an almost-contact configuration and that the primary which fills its Roche lobe is transferring mass through the inner Lagrangian point $L_{1}$ onto the surface of the secondary. This process results in a hot spot on the trailing side of the secondary and thus additional light near the first light maximum. Our photometric analysis can reproduce the observed light curves successfully by adding such a hot spot on the surface of the secondary. As for the variation of light curves, it can be explained using the idea that the mass transfer changes from strong to weak or vice versa, and even stops sometimes, thereby producing the differences in the light curves observed by the various groups. If the primary in expanding can maintain its mass loss, the system has a semidetached configuration and mass transfer can be in progress, the light curves exhibit the O'Connell effect. Otherwise, the primary may not fill its Roche lobe, mass transfer stops, and the light curves do not show the O'Connell effect.

Combining our new solution with Yamasaki et al.'s (1984) mass function, new absolute parameters of GR Tau are derived by means of the following well-known relations, and are listed in Table 3. Here the errors for the absolute parameters are 
calculated by using the light curve fitting errors of the final photometric solution. The calculation does not account for the error of the mass function because Yamasaki et al. (1984) did not publish it. Therefore, the error margins obtained are possibly unrealistic, and the real error margins should be larger than these values.

$$
\begin{aligned}
& f(m)=M_{2}^{3} \sin ^{3} i /\left(M_{1}+M_{2}\right)^{2} \\
& M_{1}=M_{2} / q \\
& R_{1} / R_{\odot}=\left(74.55\left(M_{1} / M_{\odot}\right)(1+q) P^{2}\right)^{1 / 3} r_{1} \\
& R_{2}=\left(r_{2} / r_{1}\right) R_{1} \\
& L / L_{\odot}=\left(R / R_{\odot}\right)^{2}\left(T / T_{\odot}\right)^{4} .
\end{aligned}
$$

If GR Tau is put in the H-R diagram of Hilditch et al. (1988), it is found that the two components are above the ZAMS line, but well inside the main sequence. This suggests that they are evolved main-sequence stars.

According to Shaw (1990, 1994), GR Tau is a member of the near-contact binaries. From our result, GR Tau belongs to the subclass of V1010 Oph binaries. In addition, according to the TRO theory (Lucy \& Wilson 1979), W UMa systems undergo periodic thermal relaxation oscillations. Such an oscillation comprises a contact phase and a semidetached phase. In the semidetached phase, physical and thermal contact is broken, and the light curve of the system looks like $\beta$ Lyrae-type. The primary fills its Roche lobe, and the secondary lies within its Roche lobe. Such a semidetached binary predicted by the TRO theory is in the process of transferring mass from its primary to its secondary through the inner Lagrangian point $L_{1}$. This mass transfer does not form a gaseous ring or an accretion disk because the radius of the secondary is only a little less than the radius of its Roche lobe. The gas stream that leaves the primary at the $L_{1}$ point will collide directly with the surface of the secondary to create a hot spot. This hot spot enhances the light maximum that follows primary light minimum. Thus, GR Tau may be an example in the semidetached phase predicted by the TRO theory. Since GR Tau is an evolved system, and not in contact now, perhaps it was in contact previously. This means that GR Tau oscillates between contact and noncontact states, like V1010 Oph (Corcoran et al. 1991).
Acknowledgements. $\mathrm{Gu}$ and Chen are very grateful to $\mathrm{Mr}$. Kam-yung Choi and Mr. Samuel Tong for their kind assistance during the observations in Ho Koon Nature Education cum Astronomical Centre. Many thanks are given to the referee Dr. Drechsel for his constructive comments and suggestions for our manuscript, which greatly improved our paper. This work is supported by the Applied and Basic Research Foundation of Yunnan Province, China and the grant from the Sik Sik Yuen of Hong Kong, China.

\section{References}

Agerer, F. 1991, BAV-Mitteilungen, 59

Agerer, F., \& Hubscher, J. 1996, IBVS, 4383

Agerer, F., \& Hubscher, J. 1998, IBVS, 4562

Agerer, F., Dahm, M., \& Hubscher, J. 1999, IBVS, 4712

Agerer, F., Dahm, M., \& Hubscher, J. 2001, IBVS, 5017

Corcoran, M. F., Siah, M. J., \& Guinan, E. F. 1991, AJ, 101, 1828

Díaz-Cordovés, J., Claret, A., \& Giménez, A. 1995, A\&AS, 110, 329

Eaton, J. A., \& Poe, C. H. 1984, Acta Astron., 34, 97

Fang, M., Zhai, D., Gao, Z., et al. 1994, IBVS, 4082

Faulkner, D. 1986, PASP, 98, 690

Hanzl, D. 1990, IBVS, 3423

Hanzl, D. 1991, IBVS, 3615

Hanzl, D. 1994, IBVS, 4097

Hilditch, R. W., King, D. J., \& McFarlane, T. M. 1988, MNRAS, 231, 341

Lázaro, C., Niarchos, P., Rovithis, P., et al. 1995, AJ, 110, 1796

Lucy, L. B., \& Wilson, R. E. 1979, ApJ, 231, 502

Mullis, C. R., \& Faulkner, D. R. 1991, IBVS, 3593

Shaw, J. S. 1990, in Active Close Binaries, ed. C. Ibanoglu (Dordrecht: Kluwer), 241

Shaw, J. S. 1994, Mem. Soc. Astron. Ital., 65, 95

Wichmann, R. 1998, Nightfall User Manual

Wilson, R. E., \& Devinney, E. J. 1971, ApJ, 166, 606

Wilson, R. E. 1992, Documentation of eclipsing binary computer model, privately circulated monograph

Wunder, E., Wieck, M., Kiling, B., et al. 1992, IBVS, 3760

Yamasaki, A., Okazaki, A., \& Kitamura, M. 1984, PASJ, 36, 175

Zhang, X. B., Zhang, R. X., \& Fang, M. J. 2002, A\&A, 395, 587 